\title{
A IMPORTÂNCIA DA ANÁLISE ESPACIAL PARA TOMADA DE DECISÃO: UM OLHAR SOBRE A PANDEMIA DE COVID-19
}

\section{The importance of spatial analysis for decision-making: a look at a pandemic of COVID-19}

\author{
Phillipe Valente Cardoso \\ Prof. Assistente do Departamento de Geografia da UERJ-FFP \\ phillipevalente@gmail.com \\ Vinicius da Silva Seabra \\ Prof. Adjunto do Departamento de Geografia da FFP da UERJ \\ vinigeobr@yahoo.com.br \\ Izabela Braz Bastos \\ Graduanda em Geografia da UERJ-FFP \\ Izabelabraz.uerj@gmail.com \\ Evelyn de Castro Porto Costa \\ Mestre em Geografia da UERJ-FFP \\ evelynportocosta@yahoo.com.br
}

Artigo enviado para publicação em 24/04/2020 e aceito em 30/04/2020

DOI: $10.12957 /$ tamoios.2020.50440

\section{Resumo}

A Geografia tem a possibilidade de contribuir com as crises de diferentes maneiras, permitindo realizar variadas leituras do problema e subsidiando o seu gerenciamento. No caso da COVID19, pandemia enfrentada por grande parte dos países de quase todos os continentes, é possível encontrar situações em que a Geografia tem dado importante suporte. No âmbito do geoprocessamento é possível realizar análises espaciais que ajudam na compreensão da complexidade do problema, associando a outras variáveis espaciais que podem agravar o quadro, além de avaliar tendências futuras. Este artigo tem a pretensão de fazer reflexões acerca da importância das geotecnologias para as análises de fenômenos, bem como abordar a utilidade das análises espaciais para tomadas de decisões. Como exemplo empírico, é apresentado o mapeamento dos casos de COVID-19 no município de São Gonçalo - RJ e seus desdobramentos.

Palavras-Chaves: COVID-19; pandemia; geoprocessamento; análise espacial.

\begin{abstract}
A geography has the possibility to contribute to crises in different ways, allowing to carry out different readings of problems and subsidize their management. In the case of COVID-19, the pandemic faced by most countries on almost all continents, it is possible to find situations in which geography has given important support. In the scope of geoprocessing, it is possible to perform spatial analyzes that help in understanding the problem, associating other spatial variables that may aggravate or frame, in addition to assessing future trends. This article intends to reflect on the importance of geotechnologies for analyzing phenomena, as well as addressing the use of spatial analysis for decision making. As an empirical example, it is presented or mapped the cases of COVID-19 in the municipality of São Gonçalo - RJ and its developments.
\end{abstract}

Keywords: COVID-19; pandemic; geoprocessing; spatial analysis. 


\section{Introdução}

Em 30 de janeiro de 2020, a Organização Mundial de Saúde (OMS) declarou que a COVID-19, doença causada pelo vírus da Síndrome Respiratória Aguda Grave do Coronavírus 2 (Sars-Cov-2), se configurava como uma Emergência de Saúde Pública de Importância Internacional, ou seja, o nível de alerta máximo emitido pela entidade em seu Regulamento Sanitário Internacional. Em 11 de março de 2020, a COVID-19 foi alçada ao nível de pandemia, já que os casos da doença já se comprovavam em inúmeros países, de praticamente todos os continentes.

Uma das pandemias considerada a mais severa da história da humanidade foi a gripe espanhola, causada pela virulência incomum de uma estirpe do vírus Influenza A, do subtipo H1N1. Especialistas apontam que esta foi a maior e mais devastadora das doenças que grassaram no século XX - infectou mais de seiscentos milhões e vitimou entre vinte e quarenta milhões de pessoas em todo o mundo, em um curto espaço de tempo (REID et al., 2001).

Segundo a Organização Pan-Americana de Saúde (OPAS), esta seria a sexta vez na história que se declara este tipo de emergência. Nas outras situações, a Emergência de Saúde Pública de Importância Internacional foi declarada em razão da pandemia de H1N1 (2009), disseminação internacional de poliovírus (2014), surto de Ebola na África Ocidental (2014), vírus zika (2016) e surto de ebola na República Democrática do Congo (2018).

Um dos aspectos que mais causam preocupação por parte das autoridades médicas é colapso dos sistemas de saúde das diferentes regiões por onde passa a doença. Esse colapso é provocado por: aumento exponencial do número de pessoas contaminadas; alto grau de contaminação dos profissionais de saúde envolvidos diretamente com os pacientes; longo tempo de tratamento dispensado aos pacientes mais graves; e pelo grande número de pacientes que necessitam da utilização de unidades de tratamento intensivo (UTI) e uso de respiradores artificiais.

A velocidade e capacidade disseminação da COVID-19 impõe uma série de desafios semanais, ou até mesmo diários, à saúde pública, dificultando a tomada de decisão por parte das autoridades em suas diferentes esferas de governo. O que se observa é uma corrida, em que se coloca de um lado a Ciência e a busca por tratamentos ou outros tipos de soluções que reduzam a curva de contaminação da doença e do outro o aumento exponencial de pessoas contaminadas e internadas em UTI. Este aspecto é potencializado 
$\overline{\text { pela possibilidade de contaminação por pessoas que ainda estão na fase inicial da doença, }}$ ou até mesmo por aqueles que possuem o vírus, mas não apresentam ainda seus sintomas (assintomáticos).

Diante de tantas novidades e incertezas, as únicas decisões que se tornaram praticamente consensuais na comunidade científica internacional é o isolamento social, adoção de medidas de higiene básica, o uso de equipamentos de proteção (máscaras e luvas) e aplicação de testes para identificação das pessoas contaminadas. No entanto, muitos problemas se colocam diante da contenção da crise, mesmo em situações em que se dispõe de todas estas possibilidades. Boa parte destes problemas se originam na dificuldade em compreender a dinâmica espacial da doença em escala local. E é nesse aspecto que reside a maior contribuição do geoprocessamento para a saúde pública.

A Geografia e suas diferentes áreas do conhecimento podem contribuir na organização e leitura de uma série de aspectos socioespaciais que podem subsidiar tomadas de decisão, que vão desde adoção de estratégias para proteção de grupos sociais vulneráveis e desprivilegiados, até na construção de cenários pós crise sanitária. Cada área do conhecimento geográfico, com sua especificidade, apresenta um valioso leque de possibilidades de leitura do problema. E isso não é diferente quando fazemos uso das ferramentas disponibilizadas pelo Geoprocessamento.

O Geoprocessamento é a área do conhecimento que envolve um conjunto de metodologias e (Geo)tecnologias voltadas para aquisição, armazenamento, processamento e representação de dados e informações espaciais. Deste conjunto de ferramentas, podemos destacar os Sistemas de Informações Espaciais (SIG), que dentre outras funções, possibilita o armazenamento e organização de dados espaciais, busca por informações a partir de ferramentas de seleção, análises espaciais e geoestatísticas, construção de cenários e outros.

Carvalho et. al (2000) destacam a capacidade dos Sistemas de Informação Geográfica (SIG) na análise da distribuição espacial dos casos de contaminação, evidenciando as disparidades espaciais que levam à delimitação de áreas de risco para mortalidade ou incidência de eventos mórbidos. As autoras destacam ainda o grande potencial dos SIG na avaliação dos sistemas de saúde, possibilitando a análise da distribuição espacial de serviços de saúde, planejamento e otimização de recursos de saúde, estudos de acessibilidade e utilização de serviços de saúde. Além disso, podemos realizar também a análise do fluxo de pacientes, sendo possível definir as áreas de onde provém a demanda que busca determinados recursos de saúde. 
Sendo assim, o objetivo deste artigo é chamar atenção para as possibilidades de utilização da Cartografia e o Geoprocessamento para geração de subsídios capazes de ajudar a tomada de decisão por parte de gestores, movimentos sociais e outros grupos sociais contra a disseminação da COVID-19. Além disso, discutiremos o potencial da linguagem cartográfica e sua eficácia no diálogo com a população, a partir do exemplo de mapeamento da doença no município de São Gonçalo.

\section{A importância das Geotecnologias para análise de fenômenos espaciais}

Podemos afirmar que o uso das geotecnologias é de grande relevância para estudos em geografia, uma vez que possibilita diagnósticos eficientes, propõe soluções de baixo custo e cria alternativas otimizadas para as questões enfrentadas diante das mudanças aceleradas que observamos atualmente.

Além disso, a falta de recursos financeiros e de profissionais, verificada nos diversos órgãos que tratam da questão ambiental, coloca o uso das geotecnologias como a mais relevante fonte de aquisição e manipulação de dados, e em muitas vezes, a única para os estudos socioeconômicos, de saúde ou ambientais. Também percebemos que a necessidade de manipulação de grandes volumes de variáveis tornou imprescindível o uso das geotecnologias para o acompanhamento das modificações espaço-temporais que ocorrem no mundo.

As técnicas específicas ao estudo do comportamento espacial dos objetos e fenômenos dispostos na superfície terrestre, como no caso das ferramentas de geoprocessamento, permitem a interligação de diversas ciências voltadas para estudos desta natureza. A necessidade de se trabalhar todos estes conjuntos de dados, de naturezas e fontes diversas, possibilitando análises de forma mais diversificada e integradora, promove uma incorporação crescente do uso das geotecnologias como suporte as análises em geografia. O uso destes instrumentos torna-se ainda mais importante quando tratamos de estudos que envolvam análises das mudanças ocorridas no espaço, onde temos uma enorme heterogeneidade de atores envolvidos em organização, e diferentes outras condições diversificando o seu uso.

Barcellos e Bastos (1996) apontam que o geoprocessamento é um poderoso instrumento a serviço da pesquisa em saúde. Atualmente, as análises espaciais são amplamente difundidas na área da saúde, especificamente na área da epidemiologia. 
$\overline{\text { Além de serem análises realizadas a baixos custos, auxiliam nas estratégias a serem }}$ aplicadas.

O uso dos SIG permitiu aos pesquisadores da área da saúde uma nova abordagem para trabalhar dados espaciais, permitindo a realização de tarefas que somente o tratamento estatístico e os pacotes de mapeamento não permitiam, contribuindo, assim para uma melhor compreensão dos problemas de saúde atuais (MEDRONHO, 1995).

Os avanços a partir dessa tecnologia permitem novas formas de análise e processamento dos dados em diferentes escalas, aumento na velocidade de processamento de grande quantidade de dados geográficos, trabalhos colaborativos, diagnósticos e prognósticos através dos modelos matemáticos, além da publicação e divulgação desses dados através dos SIG-WEB fundamentais para momentos de crise.

\section{As unidades espaciais de análise}

As ciências e/ou ramos do conhecimento que fazem uso de dados ou informações espaciais para suas análises devem trabalhar com a localização dos mesmos, basicamente, a partir de duas únicas maneiras: (1) georreferenciando-os, de forma absoluta, a partir de pares de coordenadas; (2) ou associando-os às unidades espaciais de representação e análise. A união, os municípios e estados, são considerados unidades espaciais de representação e análise, e neste caso, mais precisamente, unidades políticoadministrativas da República Federativa do Brasil (IBGE, 2010).

Além destas unidades, podemos ainda destacar a existência dos distritos, que são unidades administrativas dos municípios. Sua criação, desmembramento ou fusão dependem de Leis Municipais, que devem observar a continuidade territorial e os requisitos previstos em Lei Complementar Estadual. Os distritos podem ser subdivididos em unidades administrativas denominadas subdistritos, regiões administrativas, zonas ou outra denominação específica.

Já o setor censitário é a menor unidade territorial, formada por área contínua, integralmente contida em área urbana ou rural, com dimensão adequada à operação de pesquisas e cujo conjunto esgota a totalidade do Território Nacional, o que permite assegurar a plena cobertura do País (IBGE, 2010). Desta forma, por ser delimitada apenas para critérios operacionais, essas áreas além de sofrerem alterações a cada censo, dificultando análises espaço-temporais, necessitam de adaptações ao se trabalhar com escalas mais locais, como bairros. 
A divisão por bairros existe no Brasil apenas para as áreas urbanas, sobretudo para as grandes cidades brasileiras. Esta nem sempre é utilizada para fins administrativos, apesar de que em muitas das vezes traduz melhor a distribuição de algumas variáveis no espaço geográfico. Em duas situações não existem informações espaciais correspondentes aos bairros. A primeira delas aplica-se quando os municípios possuem subdivisão formal de bairros, mas os setores estão fora dos limites legais dos mesmos. E a segunda ocorre quando os municípios não têm subdivisão formal de bairros.

Definir as unidades de análise é fundamental para o entendimento dos fenômenos em si e dependem de critérios geográficos, cartográficos e da disponibilidade dos dados a serem utilizados. Cada pesquisa terá sua especificidade, para algumas o fenômeno a ser estudado precisará de uma unidade espacial detalhada, no qual não serão encontrados dados, sendo necessário gerá-los. Em outras, a análise só será possível através de uma multiescalaridade, isto é, o fenômeno não fica restrito a uma única unidade de análise. Desta forma, cabe ao pesquisador em um primeiro momento definir as questões de sua pesquisa para em seguida delimitar sua unidade de análise.

\section{Por que somente os números não respondem?}

O espaço geográfico e a escala de atuação do fenômeno são atores tão importantes quanto o fenômeno espacial em análise. E se tratando de uma pandemia como a do COVID-19, em que a velocidade de propagação da doença no tempo e espaço de se dá forma muito acelerada, isso fica mais evidente ainda. Em função disso, somente quantificar os números de infectados e vítimas da doença não é suficiente pra responder uma série de perguntas necessárias para as tomadas de decisão.

Vamos pensar em cenários e análise diferentes para um município e situações hipotéticas. Em todos os cenários, vamos considerar que o município em questão tem um milhão de habitantes, tem um quadro com 150 casos de pessoas contaminadas e tem 12 óbitos por COVID-19. É importante mencionar ainda que este município possui 50 bairros, distribuídos em uma área de aproximadamente $250 \mathrm{~km}^{2}$. Em todos os cenários temos o mesmo número de UTI e demais leitos disponíveis.

Devemos destacar que a análise de distribuição do fenômeno, baseada nestes cenários, só é possível graças espacialização dos dados e sua análise em ambiente SIG. Nestas situações hipotéticas, vamos considerar a espacialização dos casos em diferentes 
escalas. Na primeira análise, vamos pensar o fenômeno em escala geográfica a nível de bairro.

No primeiro cenário para análise no nível por bairro, temos a concentração dos surtos majoritariamente em uma única região. Desta maneira, $80 \%$ das pessoas infectadas se concentram em 5 (10\%), dos 50 bairros. Estes bairros são inteiramente urbanizados, com alto grau de verticalização.

No segundo cenário para análise no nível por bairro, temos uma dispersão maior dos casos de infecção, atingindo 20 bairros (40\%), dos 50 bairros. Estes bairros são inteiramente urbanizados, com baixo grau de verticalização.

No terceiro cenário para análise no nível por bairro, temos uma dispersão ainda maior dos casos de infecção, atingindo 40 bairros (80\%), dos 50 bairros. Estes bairros não são inteiramente urbanizados, com nenhuma verticalização (figura1).
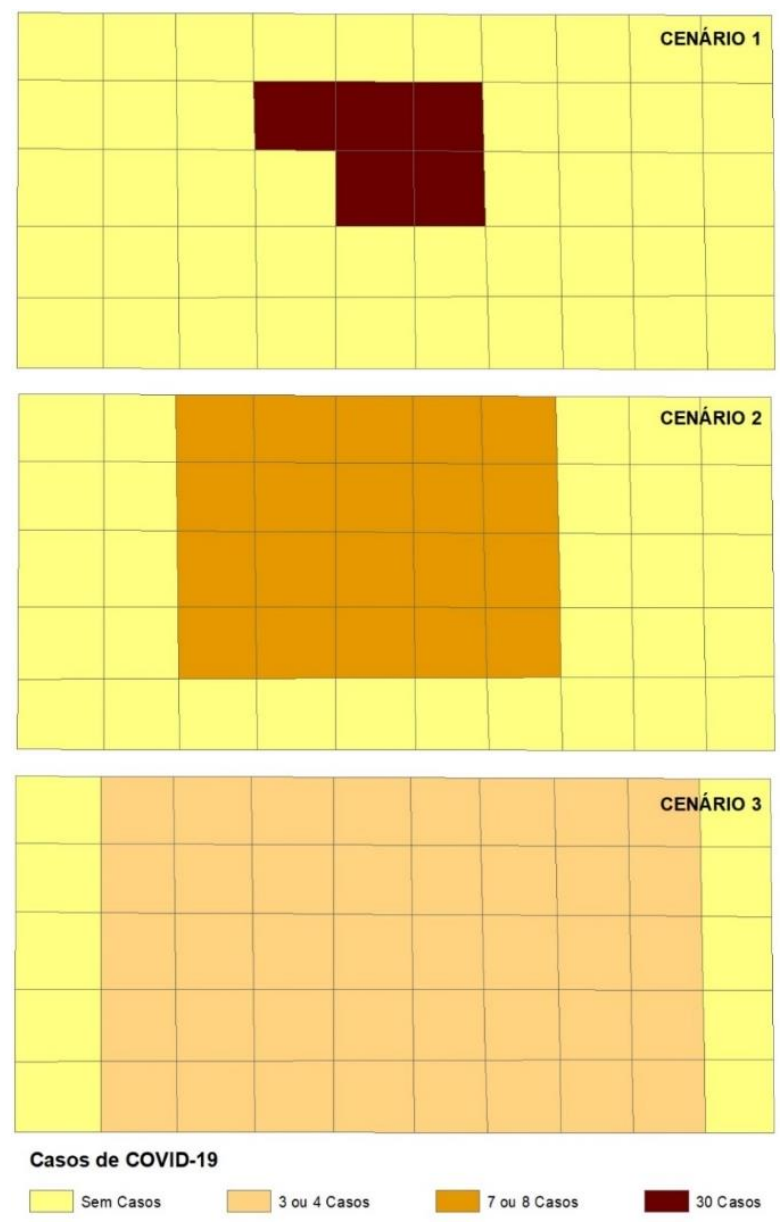

Figura 1. Cenários hipotéticos de contaminação por COVID-19

Vamos concentrar nossa análise a partir dos aspectos voltados para a contenção da doença, já mencionados na introdução deste trabalho. O isolamento social, o uso de 
materiais de proteção e a aplicação dos testes para identificação das pessoas portadoras do vírus Sars-Cov-2. Apresentaremos então algumas perguntas que abrem a possibilidade do uso de informações geradas a partir de análises espaciais para serem respondidas. Desta maneira, faremos as seguintes perguntas:

1. Deveríamos adotar medidas de isolamento social nos três cenários?

2. Este isolamento social deveria ser adotado com o mesmo rigor e ao mesmo tempo para todas as regiões do município, nos três diferentes cenários?

3. O fim do isolamento social deve ser adotado ao mesmo tempo para todas as regiões do município, nos três diferentes cenários?

4. Considerando o número limitado de equipamentos de proteção individual, deveríamos distribui-los de maneira uniforme em todo município nos três diferentes cenários?

5. Devemos definir uma estratégia única ou diferentes estratégias para testagem da população para os três cenários?

6. Nas campanhas de vacinação de doenças similares (H1N1 por exemplo), devemos definir as etapas de imunização baseando-se somente nos grupos de risco ou também na distribuição espacial da COVID-19 nos três cenários?

Se a maioria das respostas para estas perguntas considerarem estratégias especificas para os diferentes cenários, temos então a certeza de que as análises espaciais em SIG serão importantes subsídios para as tomadas de decisão. As análises ficam ainda mais complexas e interessantes se pudermos considerar variáveis socioeconômicas e de infraestrutura, tais como: densidade populacional; densidade de domicílios; habitantes por domicilio; estrutura etária da população; uso e cobertura do solo; saneamento básico; rede de transportes; localização de hospitais e postos de saúde; e outros.

Vamos agora aprofundar nossas análises para uma escala de maior detalhamento, tomando como exemplo o cenário 1 , mas com dados de localização de pessoas com COVID-19 obtidos por endereço, e não mais por bairros. Esta mudança representa não somente um aumento no nível de detalhamento da informação, mas também mudanças na forma de representação temática e, com isso, mudanças significativas das possibilidades de análise espacial (figura 2).

Como podemos perceber na figura 2, ao mudarmos a forma de representação do dado, antes associado a unidade espacial zonal "bairro", pra uma representação por pares de coordenadas (pontos) mudamos a percepção de leitura do fenômeno. Podemos agora 
inferir com mais precisão sobre outros aspectos que podem estar condicionando a expansão do número de contaminados.

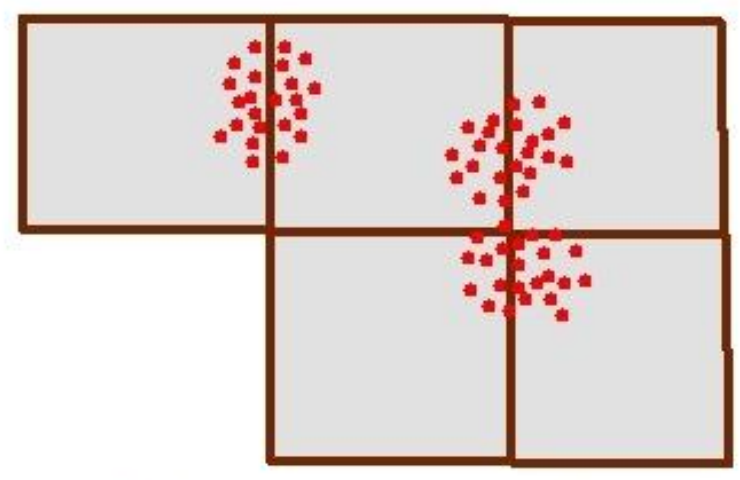

- Casos de COVID-19

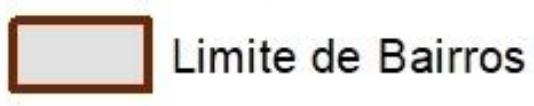

Figura 2. Distribuição dos casos de COVID-19 por endereços

Nessa nova perspectiva, podemos fazer outro tipo de análise espacial, e com isso novas perguntas, como:

1. Existe um padrão de distribuição das ocorrências? (Ex: O número de casos está concentrado?)

2. Existe dependência temática do fenômeno? (Ex: Existe hospitais no centro da distribuição dos casos?

3. Existiu algum evento correlacionado com a expansão dos casos? (Ex: Ocorreu alguma festa no centro da distribuição do fenômeno?)

Essa nova forma de ler os casos, pode subsidiar ações de mitigação. Se no centro destas ocorrências tivermos um supermercado, pode ser que o mesmo tenha que passar por ações de higienização mais severa, ou regras pra evitar aglomerações mais eficientes, pois o mesmo pode ser a causa do grande aumento de vítimas no seu entorno.

Carvalho et al. (2000) destacam que foi desta maneira que o epidemiologista inglês, John Snow, em 1854, conseguiu frear um surto de cólera no bairro do Soho, em Londres. Num período em que pouco sabíamos sobre a cólera, John Snow pontuou na planta da cidade a localização de todas as pessoas que haviam contraído a doença e percebeu um padrão espacial que evidenciava a concentração de doentes no entorno de uma bomba d'água Broad Street, atual Broadwick Street. Para resolver o problema bastou lacrar a bomba, e impedir a contaminação de outras pessoas. 


\section{A localização dos casos de COVID-19 em São Gonçalo e a importância da comunicação espacial através dos mapas}

Em março de 2020, o grupo de pesquisa em Dinâmicas Ambientais e Geoprocessamento do departamento de geografia da Faculdade de Formação de Professores - UERJ iniciou o acompanhamento através do mapeamento dos casos da COVID 19 no município de São Gonçalo - RJ divulgados através do site www.dageop.com.br e redes socais. O mapeamento ocorre por meio dos dados divulgados pela secretária municipal de saúde, aonde são monitorados os bairros com os seus quantitativos de casos.

O município de São Gonçalo, localizado na região metropolitana do estado do Rio de Janeiro possui população estimada pelo Instituto Brasileiro de Geografia e Estatística em 2017 (IBGE) de 1.090.000 habitantes, o segundo mais populoso do estado, dividido em cinco distritos e subdivididos em 91 (noventa e um) bairros oficiais e outros 18 (dezoito) bairros reconhecidos pela população em área de $249.100 \mathrm{~km}^{2}$

A prefeitura inicialmente começou a divulgar apenas dados dos casos suspeitos e confirmados da doença através de seus canais oficiais nas redes sociais, e posteriormente, passou a divulgar os casos confirmados por bairro atendendo a um pedido da população. Sendo assim, a população começou a querer respostas para perguntas: O bairro onde moro está contaminado? Quantos casos tem em meu bairro? Qual bairro com maior número de casos?

A resposta a essas questões simples podem ser feitas através de representações por tabelas e gráficos. Porém, quando perpassamos por escalas de análise buscando entender as concentrações, correlações com outras informações, fluxos, entre outras questões de um dado fenômeno é preciso espacializa-lo, mudando sua perspectiva na análise e colocando essas informações em representações cartográficas, como no caso em questão a utilização de mapas. Eles permitem refletir, criticar, comparar e compreender o espaço geográfico, fornecendo subsídios a respostas de questões mais complexas, permitindo ao usuário tanto uma aproximação do fenômeno, quanto o seu entendimento mais abrangente. Com isso, passa -se a criar e a responder outras perguntas como: Quais os bairros vizinhos aos meus tem casos? Quais as regiões do município com maior e menor incidência de casos? aonde devo direcionar estratégias para o combate a doença? Dentro da espacialização dessa espacialização, ainda é possível correlacionar dados 
$\overline{\text { através de SIG, criando representações cartográficas que concentram diferentes tipos de }}$ análises e originam verdadeiros mapas síntese.

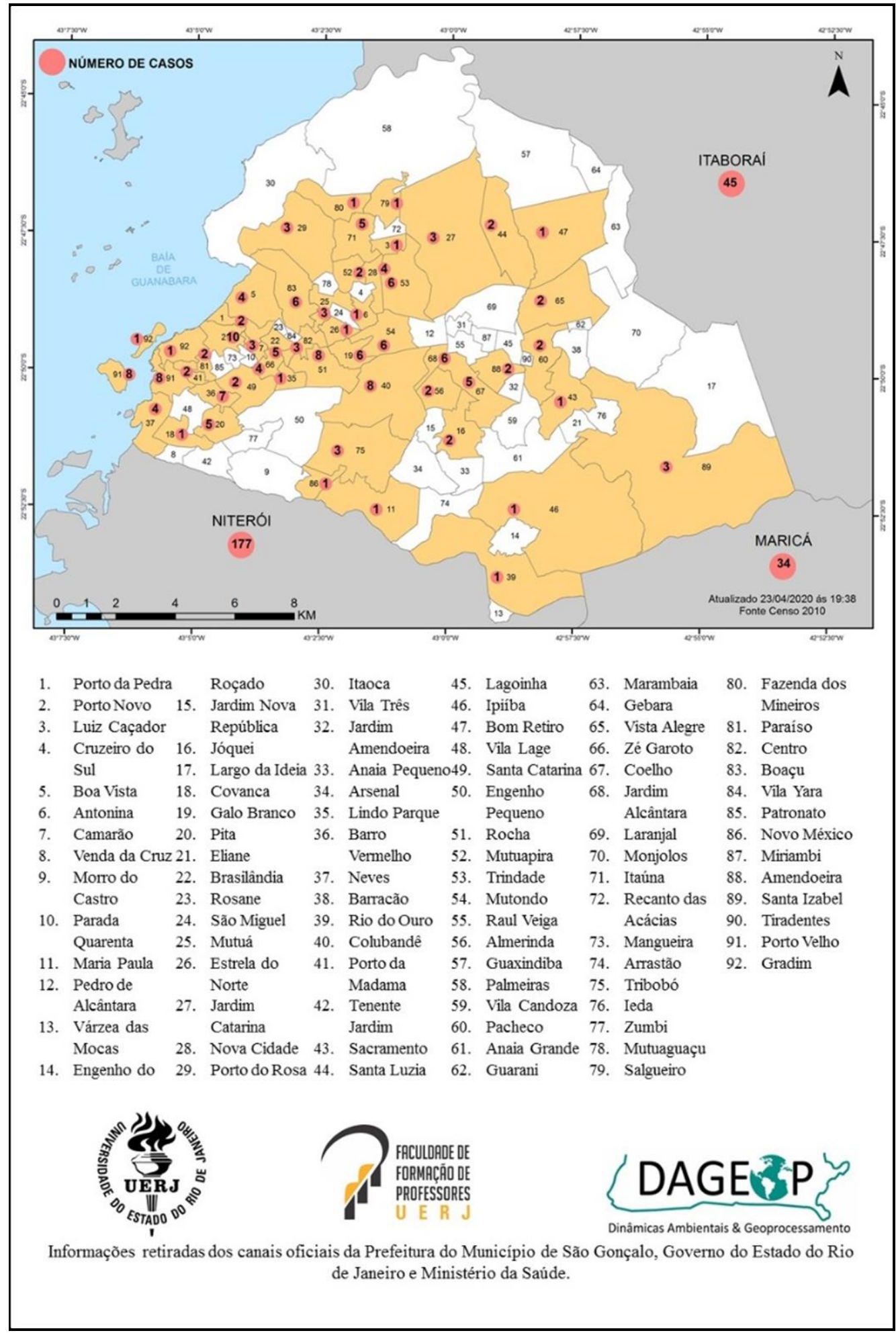

Figura 3: Casos da COVID19 no município de São Gonçalo/RJ atualizados dia

$$
23 / 04 / 2020 \text {. }
$$

Como exemplo dessas análises podemos observar a figura 3 que representa a espacialização de casos do COVID-19 no município de São Gonçalo. A presente 
representação cartográfica permite uma melhor compreensão da dispersão e concentração dos casos confirmados no município. A partir de uma análise inicial, é possível observar núcleos com os maiores números de casos nos bairros de Porto Novo, Rocha, Colubandê, sendo esses dois últimos bairros vizinhos e bairros que não apresentaram nenhum relato, mas estão cercados por casos em suas adjacências a exemplo de Recanto das Acácias e Mangueira. Essa análise topológica, ou seja, identificação de distâncias, continuidade, conectividade da relação de bairros e ao COVID-19 proporciona estratégias de combate ao vírus, permitindo a população um novo nível de percepção desse fenômeno, aproximando-os da realidade a qual seu bairro e município estão inseridas, além de possibilitar uma análise quanto ao avanço da doença para que tirem suas próprias análises e conclusões.

\section{Conclusão}

O geoprocessamento tem grande potencial para apoiar o gerenciamento de crises, como pode-se vivenciar pelo COVID-19. As análises espaciais refletem a distribuição da doença em diferentes escalas, subsidiando na tomada de decisões dos responsáveis pela saúde pública, bem como adotar estratégias mais restritivas e/ou priorizar locais com maior incidência da doença, investigando sua causa, e/ou alocando maior quantidade de insumos

Além disso, a divulgação de resultados como os mapas, auxiliam na conscientização da sociedade, dando uma nova percepção da população sobre a doença. Ao espacializar os dados, é possível ter uma outra dimensão sobre a distribuição dos fenômenos, atribuindo espacialidade aos casos e dando maiores subsídios a tomada de decisão e ao gerenciamento da crise.

\section{Referências bibliográficas}

BARCELLOS, C. BASTOS, F. I. Geoprocessamento, ambiente e saúde: uma união possível? Cadernos de Saúde Pública, Rio de Janeiro, 12.3, 389-397, 1996.

CARVALHO, M. S; PINA, M. F; SANTOS, S. M. Conceitos Básicos de Sistemas de Informação Geográfica e Cartografia Aplicados a Saúde. Organização Panamericana de Saúde. Ministério da Saúde. Brasília. 2000.

IBGE (Instituto Brasileiro de Geografia e Estatística). Censo 2000. Disponível em: http://www. https://www.ibge.gov.br/censo/. Acessado em 22/04/2020. 
MEDRONHO, R. A. Geoprocessamento e saúde: uma nova abordagem do espaço no processo saúde-doença. Rio de Janeiro: Fundação Oswaldo Cruz; 1995.

REID, Ann H.; TAUBENBERGER, J.K.; FANNING, Thomas G. The 1918 Spanish influenza: integrating history and biology. Microbes and Infection, n.3, p.81-87, 2001.

Sites consultados:

Organização Panamericana de Saúde (OPAS). Disponível em: https://www.paho.org/bra/. Acessado em 22/04/2020. 